\title{
Community Renewal with Urban Diagnosis: Bajiao Community, Shijingshan District, Beijing
}

\author{
Zhongnan YE, East China Architectural Design \& Research Institute, China \\ Hanxue WEI, Cornell University, United States \\ Chuanren LIN, East China Architectural Design \& Research Institute, China
}

\begin{abstract}
Nowadays, China's economic development has entered a new normal, and the mainline of urban construction is increasingly shifting from "incremental development of the new area" to "urban renewal of city center area". As a result, the urban renewal market is expanding rapidly and is becoming a new growth point for urban development. At the same time, with the implementation of urban renewal for the first time in the 14th Five-Year Plan, major cities across the country have released their urban renewal policies one after another, with a unified emphasis on preventing large-scale demolition and construction, while local policies have different emphases: Beijing and Chengdu place more emphasis on organic urban renewal, Chongqing encourages more market-oriented operations, and cities in Guangdong focus on further improving the existing policy system, while "determine the main body of coordination", "create a vibrant space", "retain the fire and smoke", "establish a whole life cycle management system" and "establish a whole life cycle management system" are the keywords in Shanghai's latest legislative document. In general, it seems that China's urban renewal is presenting a new situation of multi-dimensional values, multiple models, multi-disciplinary exploration and multidimensional governance, and its spatial trend is transforming from "point-level building renewal" to "piecewise overall renewal".
\end{abstract}

As the basic unit of the urban organism, community is the core point to promote the urban renewal. This paper will start from the meaning and demand of urban renewal, reveal the current dilemma and development trend of community renewal, and take the renewal planning of Bajiao community in Shijingshan District, Beijing as an example to discuss how to introduce a new way of thinking - urban diagnosis, to gain a deeper understanding of the community's operation and development patterns, to accurately diagnose the community's internal problems, to help grasp the key elememts of renewal, and then to formulate scientific and reasonable planning and renewal strategies and management policies.

\section{Keywords}

Urban Diagnosis, Urban Renewal, Community 


\section{The meaning, needs and trials of community renewal}

\subsection{Community and Community Renewal}

As one of the critical spatial carriers for industry and population, community is the medium that links different functional modules of the city. This discussion focuses on residential communities, which not only refer to the gathering and residence of a certain number of people, but also contain the social activities surrounding residential activities and various service facilities. Residential communities carry the functions of urban cultural linkages, psychological identity, and emotional belonging. The vitality of a community is an important lever to stimulate the life of a city, as it integrates community popularity, lifestyle, competitiveness, and operation mode.

The essence of community renewal is the timely restoration, remediation, and enhancement actions to compensate for the mismatch between supply and demand in the use of existing settlements during the life cycle of the buildings. Compared with new communities, existing communities in old urban areas that have been in operation for more than a decade or even decades have more active people activities, rich historical data, and strong psychological identity and emotional belonging. However, over time, due to objective constraints such as construction quality, maintenance, and operation, the original physical facilities and functional spaces of the settlements gradually decline or are in short supply, making it difficult to match the regular usage requirements of residents. On the other hand, with the development of society and economy and changes in lifestyle and technologies, the quality of the living environment and available services remain limited, making it difficult to match the rising standards and new demands. So the vitality of the community is significantly reduced. This has become the primary goal of community renewal planning.

For example, the Bajiao neighborhood in Beijing's Shijingshan District, which is known as the western gateway to the capital, is located next to the rolling hills to the west, and has a superior ecological environment and low overall development density (Figure 1). The Bajiao neighborhood in the central part of Shijingshan District includes four old neighborhoods: Bajiao North Road, Bajiao North Lane, Shougang Bajiao and Bajiao Zhongli, with a total area of about 80 hectares (Figure 2), which will be collectively referred to as the Bajiao neighborhood.

These four communities were originally built around the 1960 s as Shougang residential areas. They were once the first urbanized areas in Shijingshan District, forming a specific historical and cultural atmosphere for a long time. They have become an essential window for the Shijingshan District to showcase the image and management of the community to the outside world. However, because the area has been built for a long time, the overall appearance has become obsolete, and the existing low-quality street space, small and scarce public interaction space, and scattered and disordered parking space are no longer able to meet the demands of the community's indigenous residents and new residents.
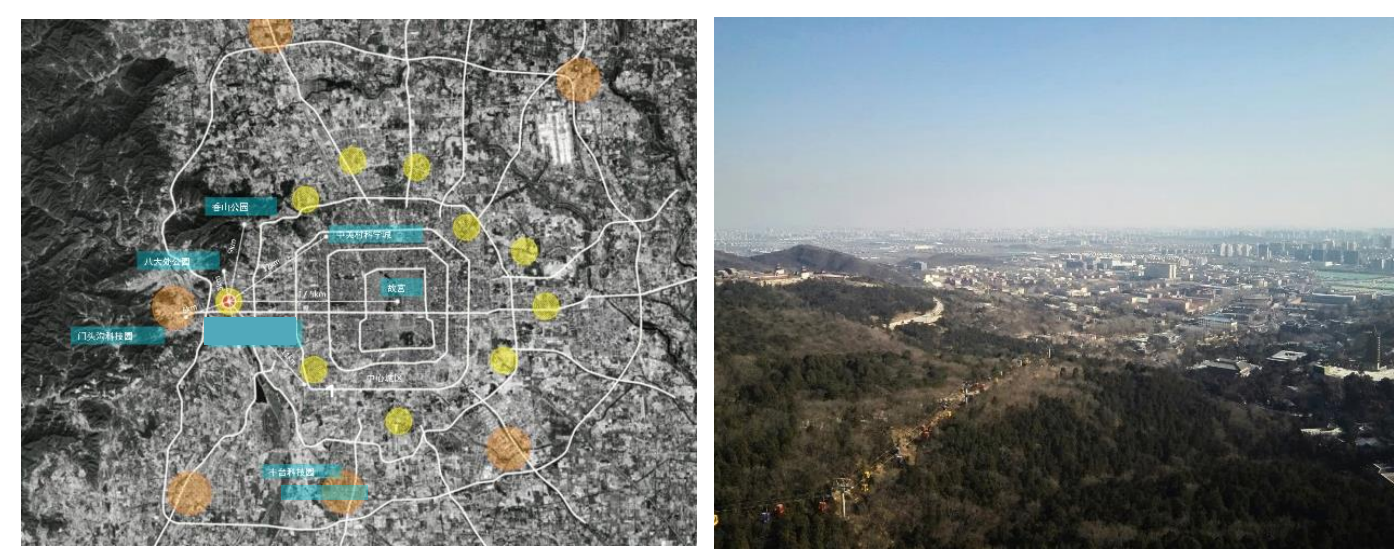

Figure 1 The location of Shijingshan District 

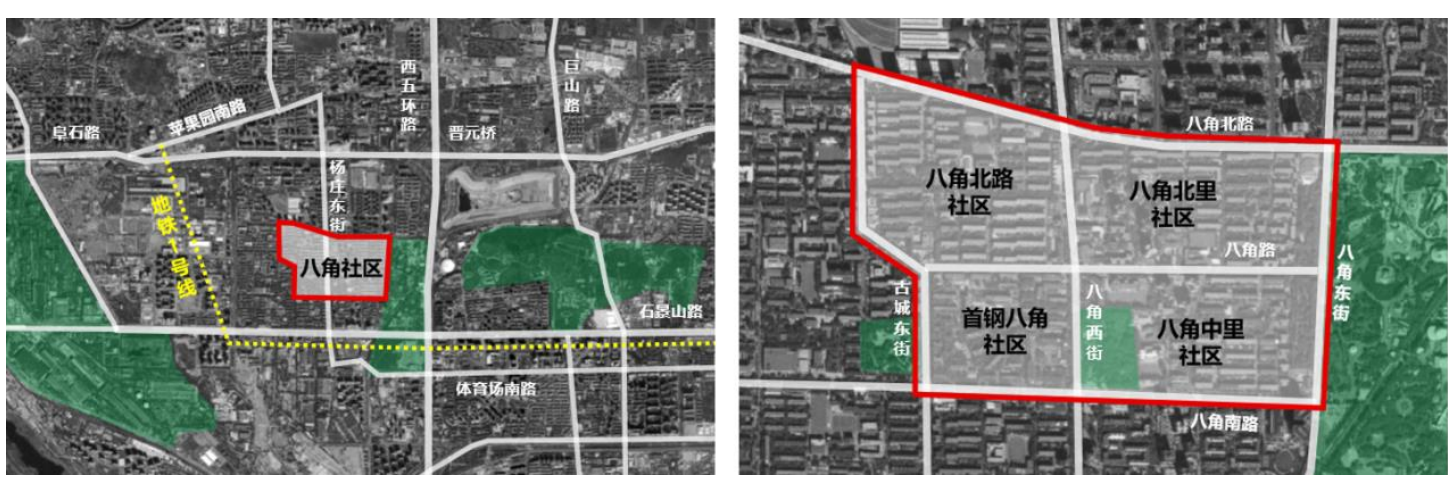

Figure 2 The location of Bajiao Community

In addition, based on the communications with the district planning department, with the guidance of Beijing's overall planning Shijingshan District is to become a national green transformation demonstration area, a green and low-carbon western capital comprehensive service area for the western Beijing, and an ecological demonstration area with the integration of landscape and culture. Thus, the specific work of creating urban gardening, road shading, community boutique, etc. is inseparable from the Bajiao community renewal. Therefore, the community of Bajiao needs "prescribing the right medicine for the right problem" to create a better community with a high quality of life.

\subsection{Traditional Community Renewal Planning Attempts}

Looking back at the past decades, the earlier community renewal practices were mainly through the following two types of paths: (1) single retention renovation, i.e., renovation of roads, building facades and other public interfaces in existing communities. This type of renovation is mostly a single special image enhancement, such as the renovation of building facades, optimization of road landscape, and replacement of street furniture, which has played a role in the improvement of urban appearance. (2) Multi-disciplinary regeneration, which aims to improve the " $\mathrm{U}$ " shaped space of the street through the cooperation of multiple fields such as the building facade, street functions, street furniture, public landscape, road traffic and municipal facilities. Facing the development demands of community renewal in the new era, it is far from enough to only renovate urban buildings and streets. Community renewal needs to start from the key "foci" of the city and the systematic factors behind them to improve urban public space based on human needs, thus creating a better place for residents.

In order to prevent and solve urban problems, China has promoted 'urban health checkups' in major cities in recent years to better support the planning work. As many as 59 cities have been identified as samples for 'urban health checkups' by 2021. In 2018, Beijing, as the first city to carry out urban health checkups, put the requirement of conducting and normalizing the urban health checkup in the Beijing Master Plan (2018-2035). It asked for regularized urban health check-ups to achieve "one health check-up per year and one assessment per five years". The Beijing Shijingshan District, where the Bajiao community is located, commissioned the Beijing Institute of Surveying and Mapping Design to prepare the "Bajiao Street Urban Health Check Assessment Report", which investigates and analyzes seven aspects, including urban population, land and buildings, comprehensive transportation, public facilities, urban environment, urban security, and urban hot spots. Based on the content, this city check-up report not only sorts out the current construction situation of the city, but also analyzes the future development space of the city. At the same time, it brings up corresponding work suggestions, offering a clear guidance for the future development of the area.

However, from the perspective of guiding communities in formulating planning strategies, the report still has shortcomings in the analysis of community operation: firstly, the object of the study is biased towards the traditional approach of focusing on physical space elements, with emphasis on morphological elements such as buildings, land and roads, while neglecting mobile elements such as people, vehicles 
and industries in urban space. Second, the analysis of the current state of community development and construction is the main focus, and the research on the history of community development and the process of problem generation in the past 5-10 years is insufficient, lacking "medical history analysis"; third, the problems are mainly stated objectively, lacking the exploration of the planning, policy, and institutional reasons behind the problems and phenomena, and the analysis of the community remains at the "inspection" level, easily leading to a superficial 'prescription' that only deals with the problem shown. In view of the limitations of urban physical examination, this study brings up the concept of urban diagnosis to expand and enrich the meaning of urban investigation and research.

\section{Intervention of Urban Diagnosis}

\subsection{Concept and meaning of urban diagnosis}

The term "diagnosis" is recognized as a bridge between basic medical science and clinical practice in the medical field and is also a key to open the door of clinical practice. For Urban Planning, theory and practice methods also consist of two important parts. The former is the summary of the laws of urban development and the explanation of planning values and methods, while the latter is the application of specific interventions in urban space. However, from the viewpoint of planning work in China and the world, there is still an obvious disconnect between planning theory and practical application. Between theory and practice, there lacks a 'diagnosis' period to connect the two parts, to make judgements on the current status and demands of the city based on the data generated in the past and present, and to provide support for the following planning practice.

Urban diagnosis, in the context of modern planning, is a process of analyzing the operation of a specific city by applying the laws, theories and experiences summarized in urban research and practice, identifying problems and exploring their causes. The diagnostic idea, to a large extent, combines problems, demands, and laws, and provides more comprehensive information and analysis on the basis of urban 'physical examination'. It can guide the subsequent strategic planning recommendations. It reflects the view and method of treating cities as living organisms, which has become an important trend in solving urban problems and making urban planning regulations in recent years.

\subsection{Expansion of urban diagnosis in the era of big data}

Since the 21st century, the rapid development of new technologies, represented by the "big data, artificial intelligence, mobile internet, and cloud computing" (1), has brought revolutionary changes to the production and life of modern people. Through the acquisition and processing of big data on the Internet, various new types of data with different data sources, accuracy levels, and data models are widely used in the urban field, providing unprecedented information support for urban problem judgment and research.

For urban diagnosis, at the technical level, multi-source big data provides a new perspective for observing and studying cities. Compared with traditional urban research, which relies more on field observation, statistical data and sample survey, multi-source big data can present development patterns more clearly by comparing data in different time sections; on the other hand, it can provide fine-grained information to the individual behavior, the trajectory of each vehicle, and the physical environment of each building. It provides a data basis and technical support for systematic and quantitative diagnosis standards. By analogy with medical diagnosis, multi-source big data provides planners with tools such as "microscope", "X-ray" and "MRI" to observe the city. In addition, compared with the side effects of clinical medical data acquisition on samples, the access to urban data is more convenient and can even achieve all-round realtime monitoring, which is significant in supporting the urban diagnosis system and promoting changes. 


\subsection{Application of Urban Diagnosis in Community Renewal}

The hierarchy of urban diagnosis includes the overall level, the system level and the district level, and the needs for diagnosis at different levels of planning or policy making have different focuses. The diagnosis of community belongs to the district level. The focus of the diagnosis is not related to the systematic diagnosis, because the district itself is only a component of the city. The emphases are on the combination of the positioning and characteristics of the district itself to judge whether its function is in line with its role setting, and then to carry out the diagnosis at the medium and micro scales.

From the viewpoint of ideology and methodology, urban diagnosis is influenced by the diagnostic thinking and methodology of medical science and other disciplines. It is also rooted in the existing rational thinking of modern urban planning, the planning idea of "shape and flow interaction", and the theory of 'urban life'. From the perspective of 'urban life', the objects to be examined and diagnosed in a city can be divided into eight major systems: information and decision making, energy, water resources, material circulation, atmosphere, production, life and balance maintenance, each of which is relatively independent and interrelated. The residential community is the main component of the urban living system. According to the element composition and diagnosis system of the 8 systems, community vitality is the main measure of living system diagnosis, and its main diagnostic indicators include four major aspects, such as crowd concentration, public service supply, public space usage and community activity participation. Combining multi-source big data and the corresponding comprehensive urban diagnostic model(2) to quantify and compare the current and historical operation of these elements and to find the parts that need improvement are the keys to the diagnostic work in community renewal planning (Figure 3).

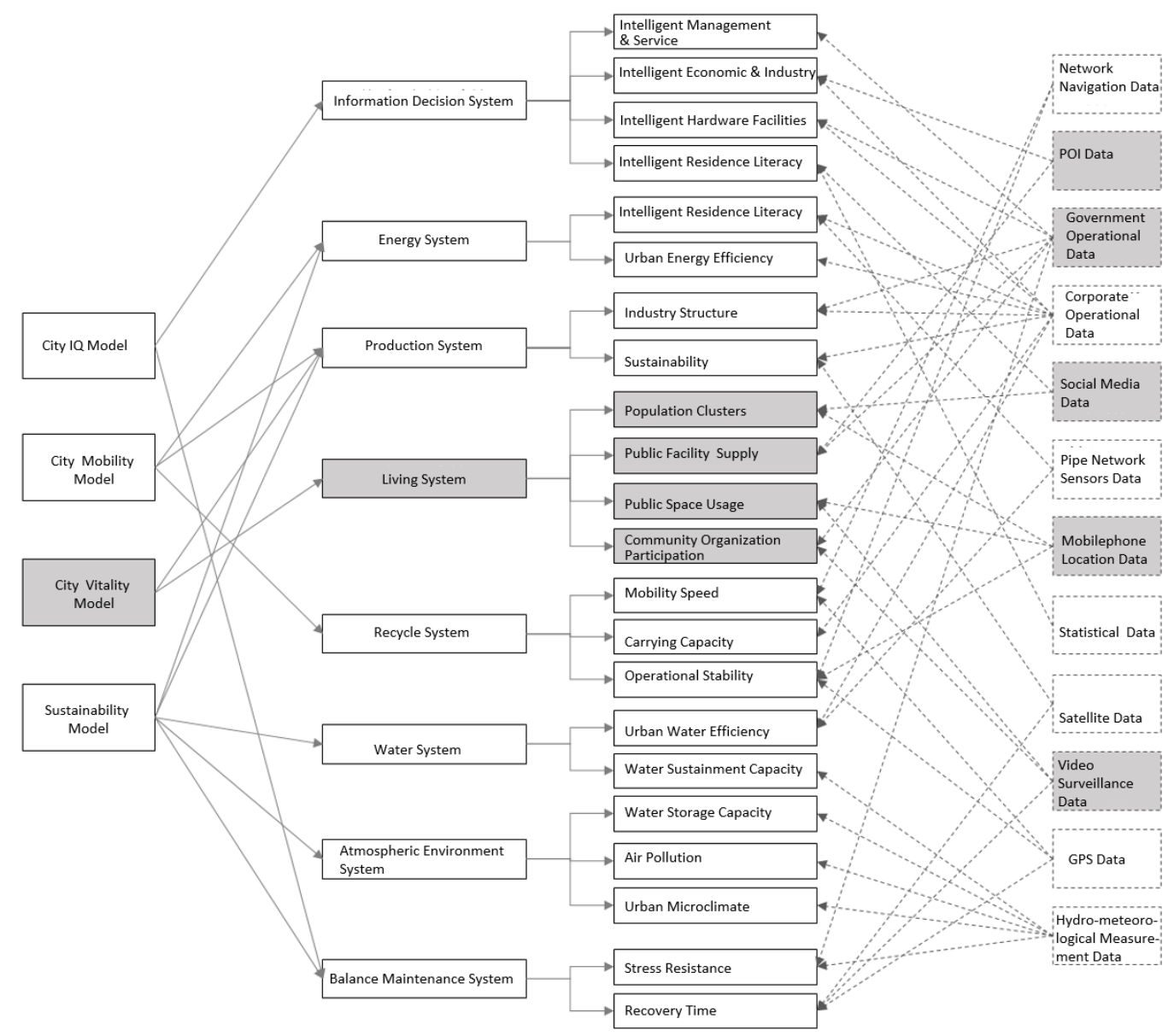

Figure 3 Diagnostic model of community (living system), main examination indicators and data sources 


\section{Diagnostic application in the regeneration planning of Bajiao community in Shijingshan District, Beijing}

\subsection{Working framework}

The urban renewal of the Bajiao community in Shijingshan District, Beijing, introduced the ideological method of urban diagnosis. Based on the urban examination report, it further helped the community planning to better grasp the key contradictions in the complex system and formulate scientific and reasonable planning, as well as renewal strategies and management policies. The work process was divided into the following four steps: first, we conducted an urban diagnosis of the planning area, verified the current problems reflected in the research report through the results of the diagnosis, and traced the "medical history" to sort out the implementation of the community renewal work and the feedback of the effect; at the same time, we used a combination of traditional research data and multi-source big data to carry out a comprehensive study of the overall community. The next step was to draw a diagnosis of the Bajiao community through the integration of all the information; finally, a targeted community renewal planning strategy was formulated based on the diagnosis.

\subsection{City Consultation}

(1) Subject of consultation

In view of the characteristics of the Bajiao community, the consultation was conducted in five groups of people, namely the government officials in charge of Shijingshan District, the person in charge of Shijingshan Branch of Beijing Planning Commission, the person in charge of Bajiao Street, the residents of the community, the property management staff, and the staff of district services and facilities, etc. Different consultation methods and focuses were adopted according to the characteristics of different groups of people. By sorting out the information from the consultation, it can be found that the issues with the highest concern for the development of the area were focused on parking lots, public space, pedestrian environment, activities for the elderly, and illegal buildings.

(2) The main complaint

In the communication with the relevant government officials in charge of the district, we learned that the main demand of organizing the Bajiao community renewal planning was to improve the lower quality community streets and public space (Figure 4) in order to meet the standard of building an ecological and livable demonstration area with mountain and water culture integration in Shijingshan District. It was crucial to pinpoint the needs of community users for public space and service facilities, sort out the elements that negatively affect the quality of community space, identify the specific types and scales of facilities that were lacking in the community, and propose corresponding regeneration strategies. This claim was clearly described, in line with the field research findings, providing a clear guidance.
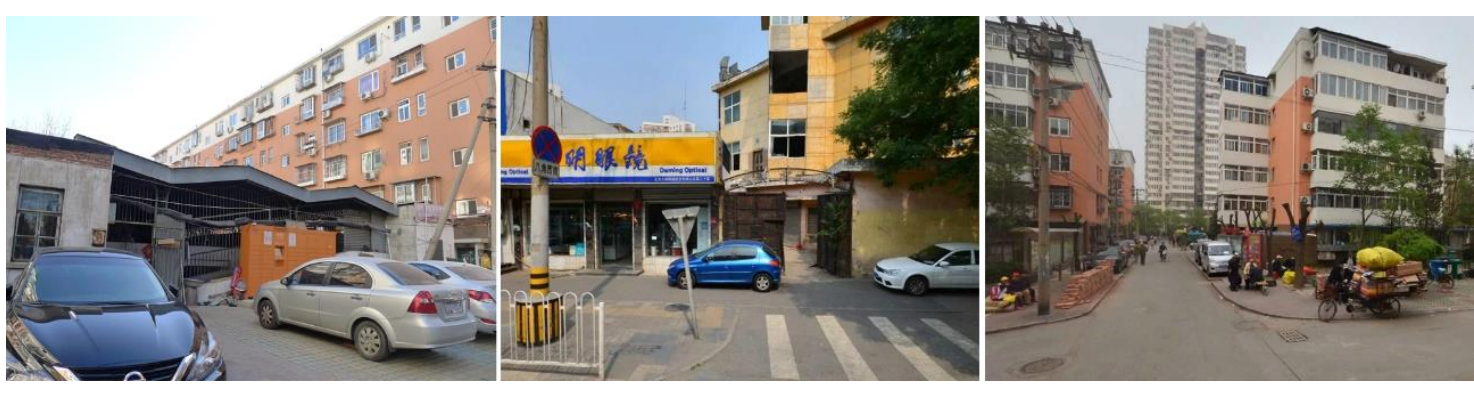

Figure 4 Public space and street quality of the base

(3) Medical history analysis: history of transformation and implementation

Renewal work was carried out in the area three years ago. At that time, the focus was mainly on the 
beautification of street facades along the streets and the necessary traffic organization, but the overall effect on the area was very limited after the completion of the renovation work. After field research and interview assessment, the main reasons for the limited effect include three points: First, the efforts were limited to the beatification of the street facade, wall painting, and billboard rectification work. The street space lacked multi-level, three-dimensional consideration. There was also lack of consideration for the ground material, green landscape, public space, and street furniture. Second, the renewal and transformation were not combined with human activities. Third, the investment was too homogeneous. Almost every street and every building were upgraded, but there was a lack of concentrated highlights, which made it difficult to be perceived and recognized by the public. The area lacked representative images and activity cores (Figure 5).
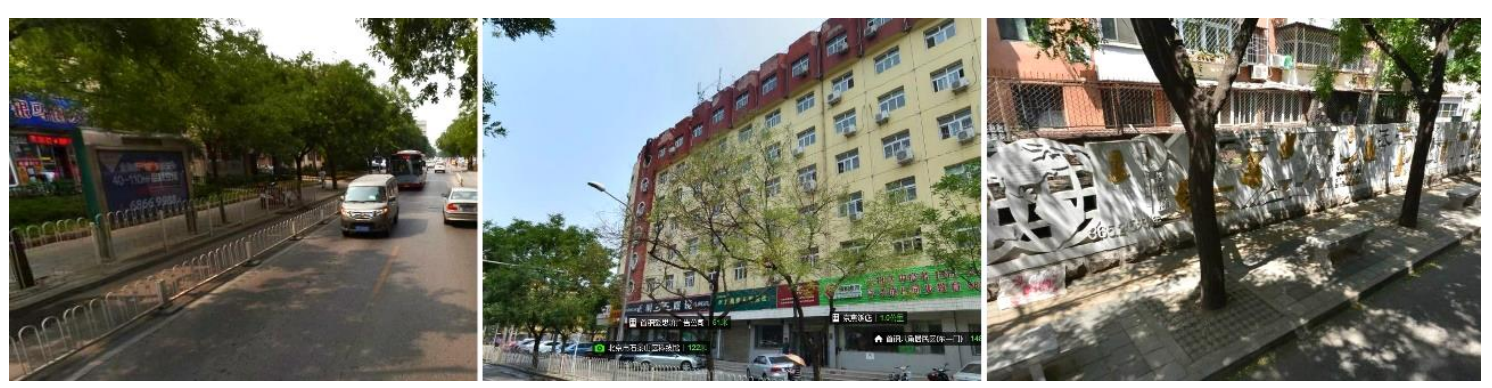

Figure 5 Separation fence, building façade and fence after the last round of remediation

\subsection{Multi-source data-based inspection of urban living systems}

According to the main functions of the Baijiao community, the corresponding indicators of the urban living system were examined. Based on the Urban Vitality Diagnostic Model (3), the community vitality examination had four main indicators, including crowd concentration, public facility supply, public space use, and community activity participation. At the same time, based on the indicators, a more in-depth analysis of their spatial and temporal changes was carried out. The main data used include not only traditional data such as questionnaire data and field research data, but also multi-source big data such as web map heat data, POI data, and video surveillance data.

\section{(1) Community Vitality}

The community vitality of the Bajiao community was diagnosed according to the urban vitality diagnostic model. The results showed that the regional single-day average vitality value was 27.5 , which was moderately low compared with similar regions in China, and even slightly lower than other neighboring communities (Figure 6), reflecting a certain lack of vitality in the region. It was necessary to further examine the four indicators related to urban vitality.

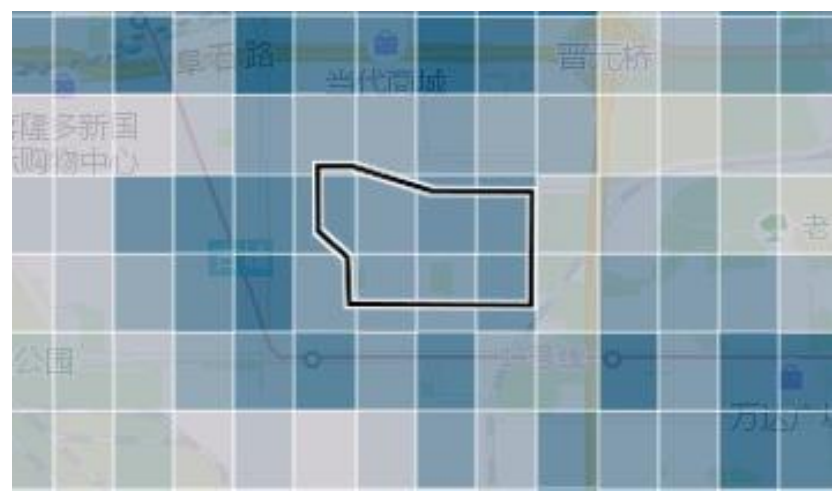

Figure 6 Results of Bajiao Community Vitality Diagnosis (500m*500m grid)

(2) Crowd Clustering 
The heat data provided by Tencent's "Yitravel" was used to check the crowd concentration in the Bajiao neighborhood. The main components of the check included the size and density of the crowd gathering throughout the day, and the change of crowd gathering during the day. Based on the vectorization of this heat map data, the study calculated the average value of crowd clustering throughout the day (Figure 7) and divided the corresponding clustering into seven levels, with values ranging from 0.35 to 11.5 and higher heat values representing higher crowd density. The overall single-day average heat value of the Bajiao community was 3.70 , which is a medium level in comparison with similar areas in China. Considering the smallest unit suggested for community management and planning strategy, the whole planning area was divided into 5 plot units based on factors such as roads, property rights, and property management. The population agglomeration scale of each unit by time was calculated based on the area size and heat level (Fig. 8, Figure 9, Figure 10).

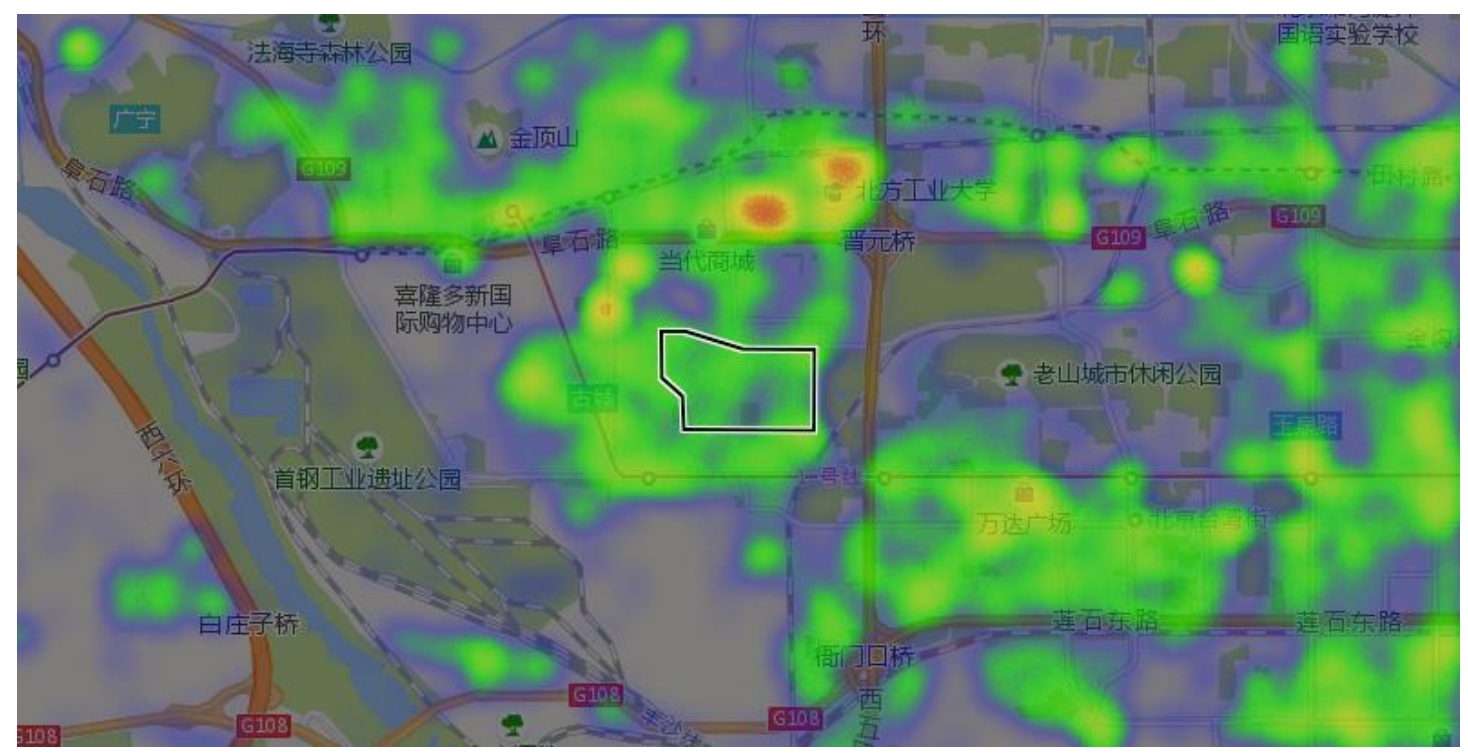

Figure 7 Heat map of regional crowd gathering (average value throughout the day)

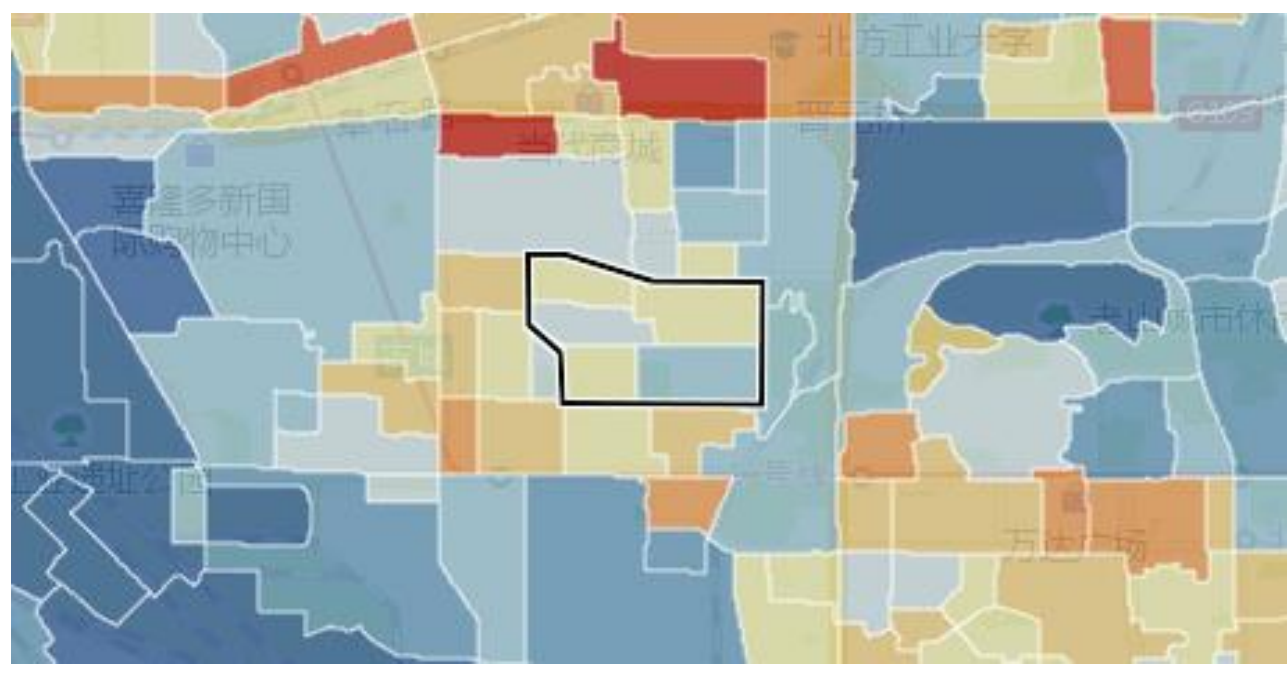

Fig. 8 Heat value of population clustering in each plot unit (8:00) 


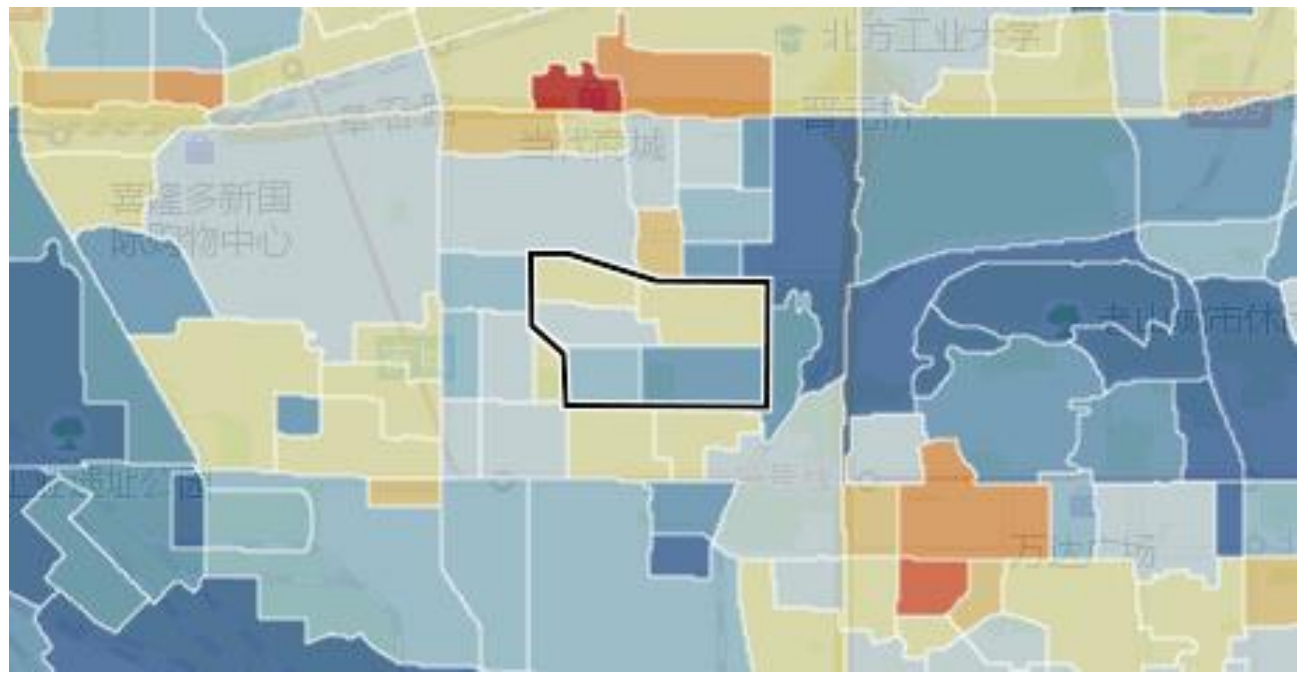

Figure 9 Heat value of crowd gathering in each plot unit (15:00)

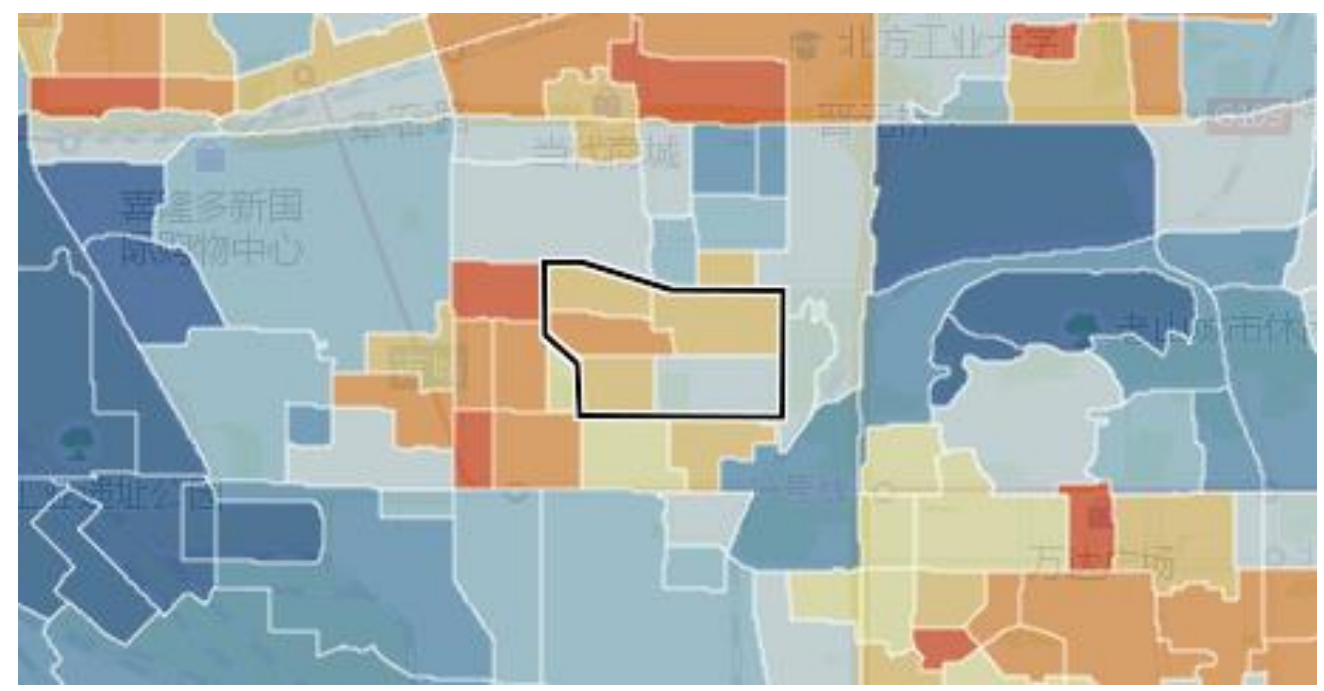

Figure 10 Heat value of crowd gathering in each plot unit (21:00)

(3) Public service supply

The scale of public service supply was obtained by the number of specific POIs within 100 meters around the point, and the richness of supply was calculated by the information entropy of POI categories. Based on the two, a composite index of public service supply level was established to directly reflect the overall situation of public service supply in the region.

In selecting the public service facilities to be examined, a total of 15 facilities in four categories were selected (Table 1). The selection criteria were based on two main considerations: first, the area belongs to an old community in an urban center, and the public services needed were mainly for daily life in the community; second, the total area was only 80 hectares, and the facilities examined within the base are mainly at the community level, while municipal or district-level facilities, such as hospitals and schools, were studied in a larger context.

The quantitative diagnosis shows that the total number of the 4 types of facilities within the Bajiao community was 56 . The category-based information entropy is 1.78 . The overall public service facility supply level was 6.75, which is moderately low in comparison with similar areas (Figure 11). In terms of specific POI types, the supply of regional commercial and community-based services was better; the number of leisure facilities was slightly insufficient; public parking facilities had a more obvious deficiency (Figure 12). 
Table 1 Facilities selected for public service supply diagnosis and classification

\begin{tabular}{l|l}
\hline Facility Type & Facility Content \\
\hline \hline Business Facilities & Food markets, supermarkets, hardware stores, restaurants, banks \\
\hline $\begin{array}{l}\text { Community } \\
\text { Service Facilities }\end{array}$ & $\begin{array}{l}\text { Community activity center, elderly service center, medical station, postal } \\
\text { service, police station }\end{array}$ \\
\hline Leisure facilities & Coffee cafe, chess and card room, gym, internet cafe \\
\hline Parking facilities & Open to the community of public parking / storage \\
\hline
\end{tabular}

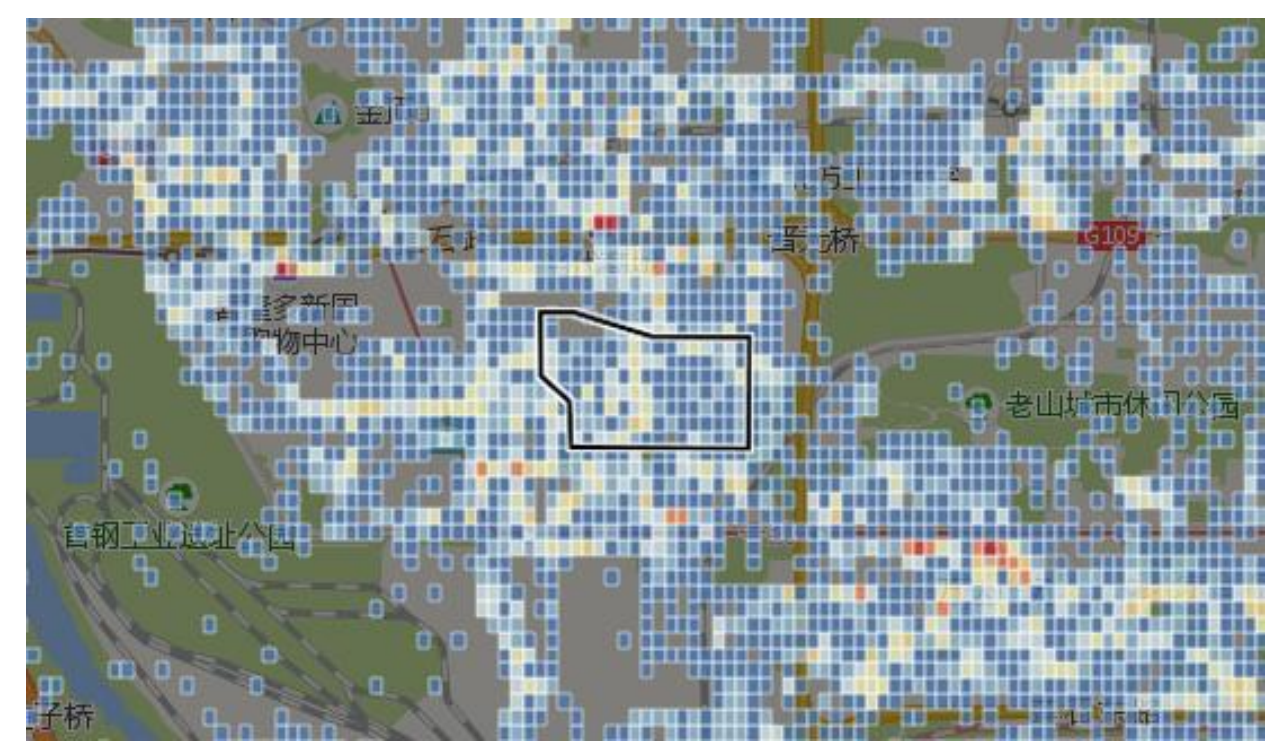

Figure 11 Regional community public service facility supply levels
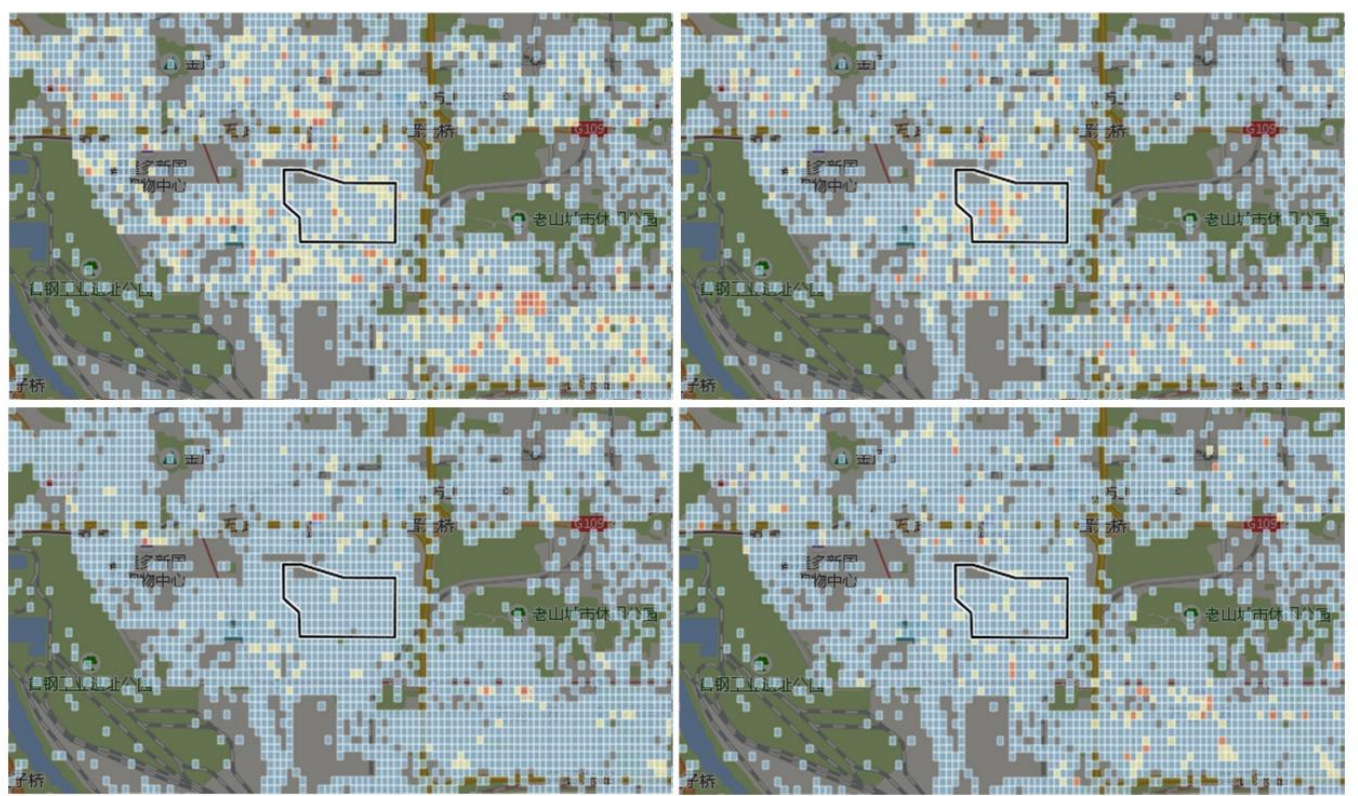

Figure 12 Major types of facility supply levels 
(Top left: commercial facilities, top right: community service facilities, bottom left: parking facilities, bottom right: recreational facilities)

(4) Public space use

Regional public space usage is mainly quantified by the number of people and duration of outdoor activities in each block unit. The project used a combination of community video data and field observation research. Firstly, the overall intensity of regional public space usage was calculated. After dimensionless processing, the average value of the whole day was 36.7 , which is a medium-low level in comparison with similar regions. This reflects the low level of public space usage.

In addition, the study further tracked the public space usage of each area unit in and around the Bajiao community at different times of the day, and clustered the area units according to the intensity of use and time of day. As can be seen, the plots were divided into three main categories in terms of the active time of public space usage: first, higher activity in the morning and evening, and lower activity during the day (working hours); second, higher activity during the day (working hours), and lower activity in the morning and evening; and third, more stable activity overall. After adding the classification of the intensity of usage, the total can be divided into eight categories (Figure 13), of which the base range mainly includes four categories (Figure 14). In terms of area, the morning and evening active ones and daytime active ones accounted for almost half each. 


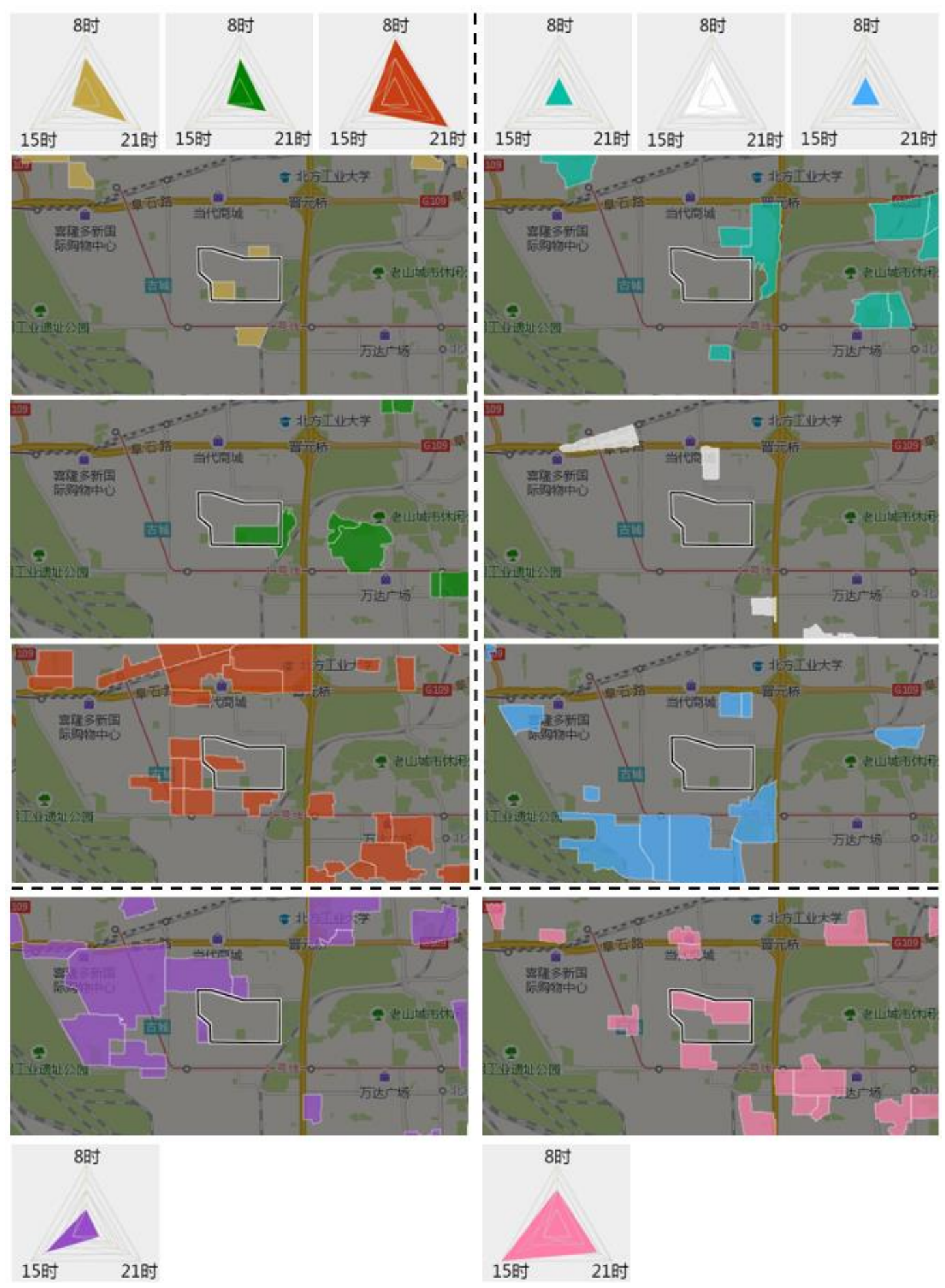

Figure 13 Clustering of plot units based on outdoor activity time and size characteristics 


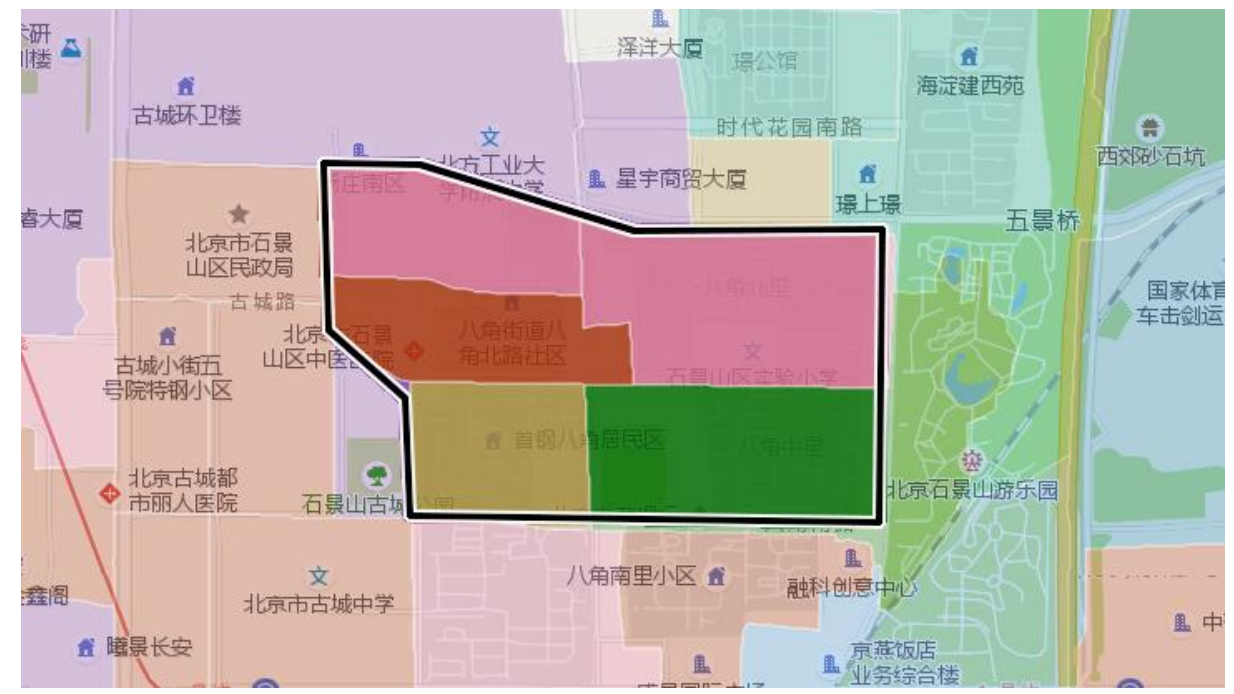

Figure 14 Classification of outdoor activity time - size of each block unit in the Bajiao community

\section{(5) Community event organization participation}

According to the data compiled from community video analysis, online questionnaires, resident interviews, and on-site research, the activity time of the population in Bajiao community, i.e. the average time per person per day for the use of public facilities and public spaces, was 1.4 hours, which was at a moderate to low level compared to similar areas in China, reflecting the low level of community activity participation. Based on the experience of similar cases, the possible reasons include: climatic reasons (cold, rainy, etc.), high proportion of working people (young adults) in the community, insufficient supply of public services in the community, insufficient scale of public spaces and lack of attractiveness of public spaces. From the meteorological characteristics of Beijing and the demographic data, the hot-summercold-winter climate and air pollution may be one of the reasons for the low participation in outdoor activities. The community's demographic structure, on the other hand, was generally balanced, with a little bit more aging problem. So, the demographics cannot explain the problem. To further search for the causes of the problem, an in-depth examination of the specific types of community activities within the area and their changes over time during the day showed that the diversity of the major community activities occurring within the Bajiao community was low, with a predominantly spontaneous type of organization and a low degree of community or commercial agency dominance. The public space did not have a significant effect on activities. In terms of activity time, the daytime (working hours) was the peak of activity intensity, and after 20:00 p.m. the usage rate of both facilities and public space decreased rapidly, reflecting the low level of participation of young people or office workers in community activities.

\subsection{Diagnostic conclusion}

According to the information from the integration of different urban inquiry objects and methods, the Bajiao community, as a typical community for workers built more than 30 years ago, has been an example of advanced community for a certain period of time after its completion. Even until today, the old residents still retain their sense of community belonging and honor. However, as time passes by, the area, whether in terms of spatial structure, urban management, traffic organization, or image and appearance, can no longer fully meet the current community's demographic structure and living needs. It is even more difficult to meet the latest development requirements of Shijingshan District, which is to build an ecological and livable demonstration area integrating landscape and culture.

From the examination of the community living system in Bajiao District based on multi-source data, the overall vitality of the area is insufficient, the scale of crowd gathering is relatively normal, while the three indicators-public service supply level, intensity of public space use and community activity participation, 
are low compared with similar areas (Table 2), meaning that the residential and working population amount of the area is reasonable, but the inadequacy of facilities, space creation, and activity organization affects the vitality and quality of life of the community, resulting in the phenomenon that a large number of residents are either unwilling to go out for activities, or choose to go to more distant places to use public facilities or carry out community activities.

Table 2 Conclusions of the Bajiao Community Living System Inspection (4)

\begin{tabular}{|c|c|c|c|c|c|c|c|c|}
\hline $\begin{array}{c}\text { Tier } 1 \\
\text { Indicators }\end{array}$ & Data & $\begin{array}{l}\text { Refer } \\
\text { ence }\end{array}$ & $\begin{array}{c}\text { Tier } 2 \\
\text { Indicators }\end{array}$ & Data & $\begin{array}{c}\text { Refere } \\
\text { nce }\end{array}$ & Tier 3 Indicators & Data & $\begin{array}{c}\text { Referen } \\
\text { ce }\end{array}$ \\
\hline \multirow{7}{*}{$\begin{array}{c}\text { Community } \\
\text { Vitality }\end{array}$} & \multirow{7}{*}{$27.5 \downarrow$} & \multirow{7}{*}{$32-42$} & $\begin{array}{l}\text { Crowd } \\
\text { Clustering }\end{array}$ & \multirow{2}{*}{$3.7 \rightarrow$} & \multirow{2}{*}{$30-50$} & $\begin{array}{c}\text { Spatial } \\
\text { dispersion } \\
\text { (Standard } \\
\text { deviation) }\end{array}$ & $4.6 \uparrow$ & $3.0-4.0$ \\
\hline & & & $\begin{array}{c}\text { (Single day } \\
\text { average) }\end{array}$ & & & $\begin{array}{l}\text { Temporal } \\
\text { dispersion } \\
\text { (Standard } \\
\text { deviation) }\end{array}$ & $3.9 \uparrow$ & $2.6-3.3$ \\
\hline & & & \multirow{2}{*}{$\begin{array}{c}\text { Public } \\
\text { Services } \\
\text { Supply Level }\end{array}$} & \multirow[t]{2}{*}{$6.7 \downarrow$} & \multirow[t]{2}{*}{$8-16$} & $\begin{array}{c}\text { Number of } \\
\text { facilities } \\
\text { (normalized } \\
\text { number) }\end{array}$ & $9 \downarrow$ & $10-20$ \\
\hline & & & & & & $\begin{array}{l}\text { Facility Richness } \\
\text { (Entropy value) }\end{array}$ & $1.7 \downarrow$ & $2.1-3.6$ \\
\hline & & & \multirow{2}{*}{$\begin{array}{l}\text { Public space } \\
\text { Intensity of } \\
\text { use }\end{array}$} & \multirow{2}{*}{$1.4 \downarrow$} & \multirow{2}{*}{$2-6$} & $\begin{array}{l}\text { Usage efficiency } \\
\text { (Personnel*hour } \\
\text { /square meter) }\end{array}$ & $25 \rightarrow$ & $19-38$ \\
\hline & & & & & & $\begin{array}{c}\text { Usage } \\
\text { Continuity } \\
\text { (\%) }\end{array}$ & $29 \downarrow$ & $21-50$ \\
\hline & & & $\begin{array}{c}\text { Community } \\
\text { Events }\end{array}$ & $38 \downarrow$ & $\begin{array}{l}50- \\
100\end{array}$ & $\begin{array}{c}\text { Event size } \\
\text { (Persons*hours) }\end{array}$ & $97 \downarrow$ & $\begin{array}{l}130- \\
520\end{array}$ \\
\hline
\end{tabular}




\begin{tabular}{|l|l|l|l|l|l|l|l|}
\hline & & & Participation & & & Activity diversity & \\
& & & & & & & \\
\end{tabular}

\subsection{Feedback on diagnostic results: developing planning strategies}

In response to the problems of public space and facilities in the diagnosis, according to the experience of past urban renewal implementation, the overly even resource input has caused the scattered and limited outcomes. On the one hand, this fails to attract the attention of community users and administrators; on the other hand, it violates the principle that activities and vitality need to be concentrated. Therefore, this urban renewal established the planning principle of "systematization, local activation, and point to area". A series of strategies have been adopted, such as the expansion of public space through comprehensive parking regulation, the creation of refined scenes to improve the quality of public space, and the construction of 15-minute living circle to complement the supporting facilities. These strategies focused on solving the most critical and urgent issues to achieve the goal of urban renewal with minimal resources and cost (Figure 15).

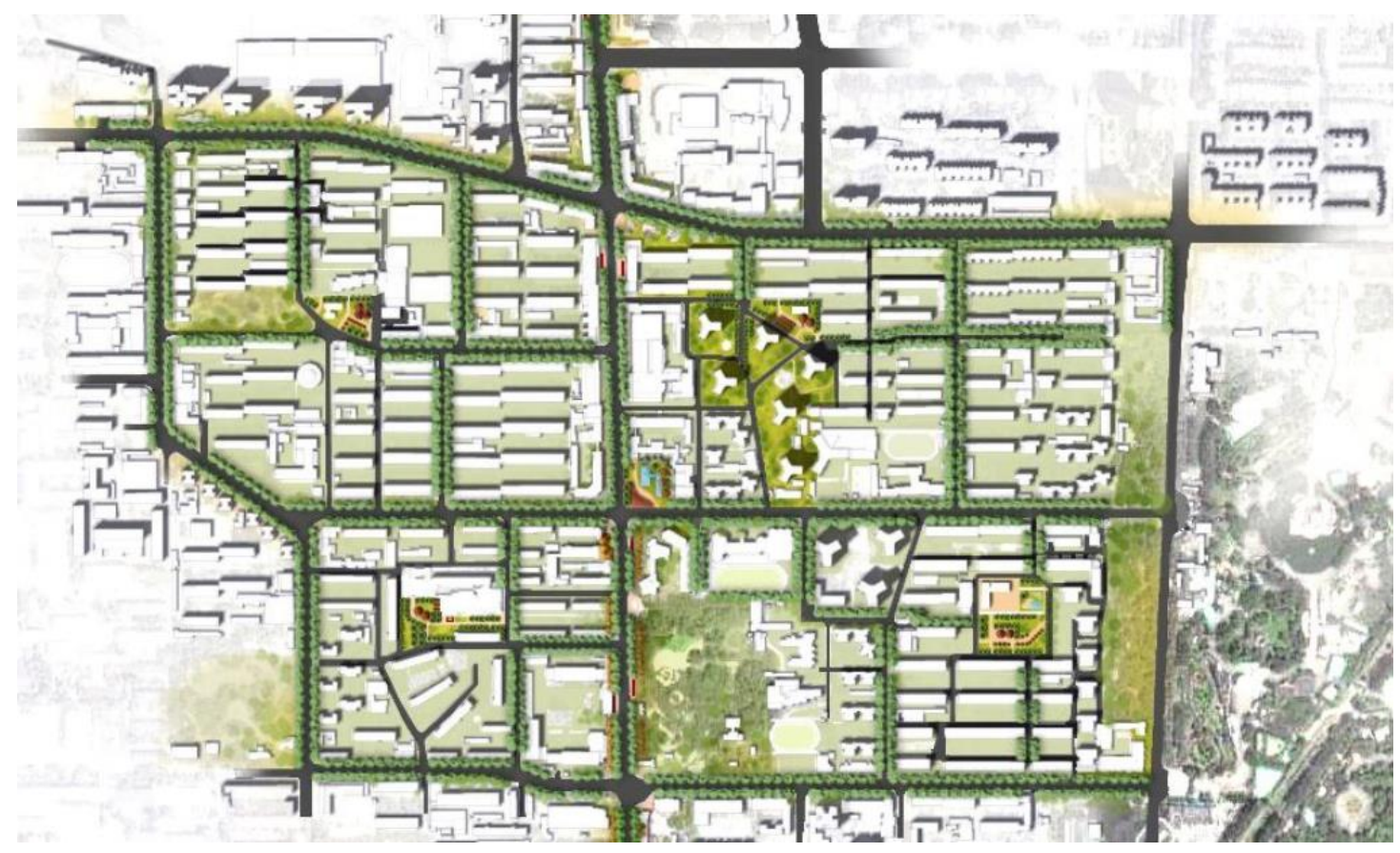

Figure 15 Bajiao Community Renewal Plan Master Plan

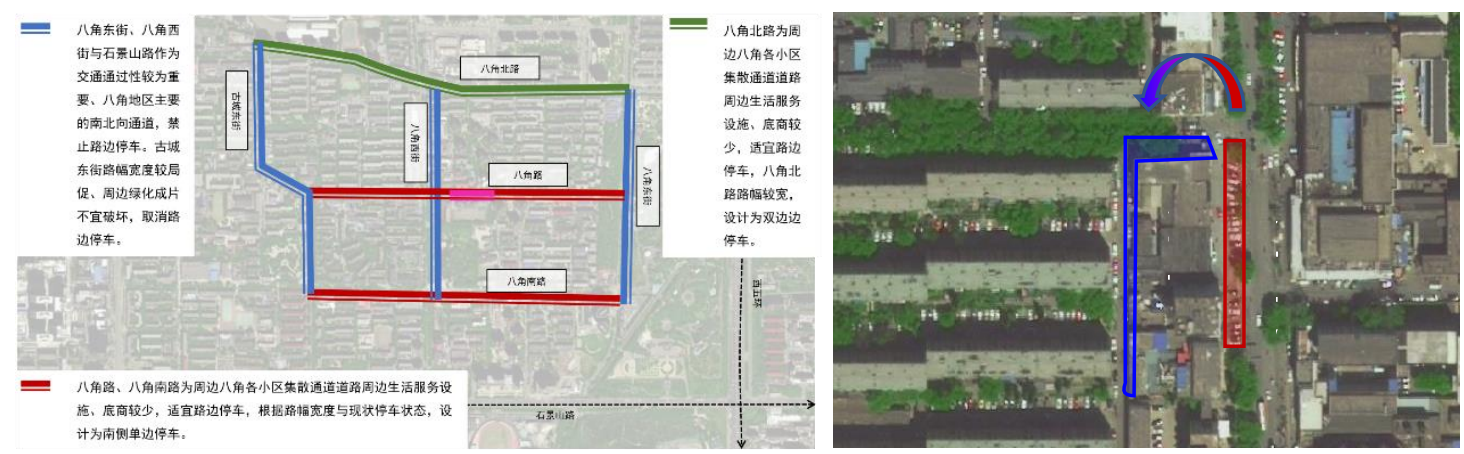

Figure 16 Comprehensive parking regulation

(1) Strategy 1: Comprehensive parking regulation to release more space for public activities 
According to the diagnosis, the main problem of the Bajiao community in terms of physical space is the lack of public space and public facilities, which is largely due to the large amount of surface parking crowding out the space. In order to solve the problem of difficult parking in the area, parking improvement was carried out in two major directions: lineary combination and local reshaping, in order to release more space for public activities.

Firstly, the roads are divided into traffic-oriented roads and life-style roads based on characteristics. Traffic-oriented roads are prohibited from on-street parking, and life-style roads are designed with legalized single-sided or double-sided parking according to the width of the road. Secondly, sidewalk occupied parking is banned to ensure the completeness of the slow traffic system. (Figure 16)

The local reshaping aims to make use of the vacant land left by the demolition of the bad building at the intersection of Bajiao West Street and Bajiao Road to create a community center in the form of "urban green steps" (Figure 17), forming a vibrant landmark for the area while making full use of the underground space to provide about 250-300 parking spaces for the community. This step will realize the integration of social parking, community management, fashion, catering, leisure, and public green space, solving as many problems as possible within a limited space.

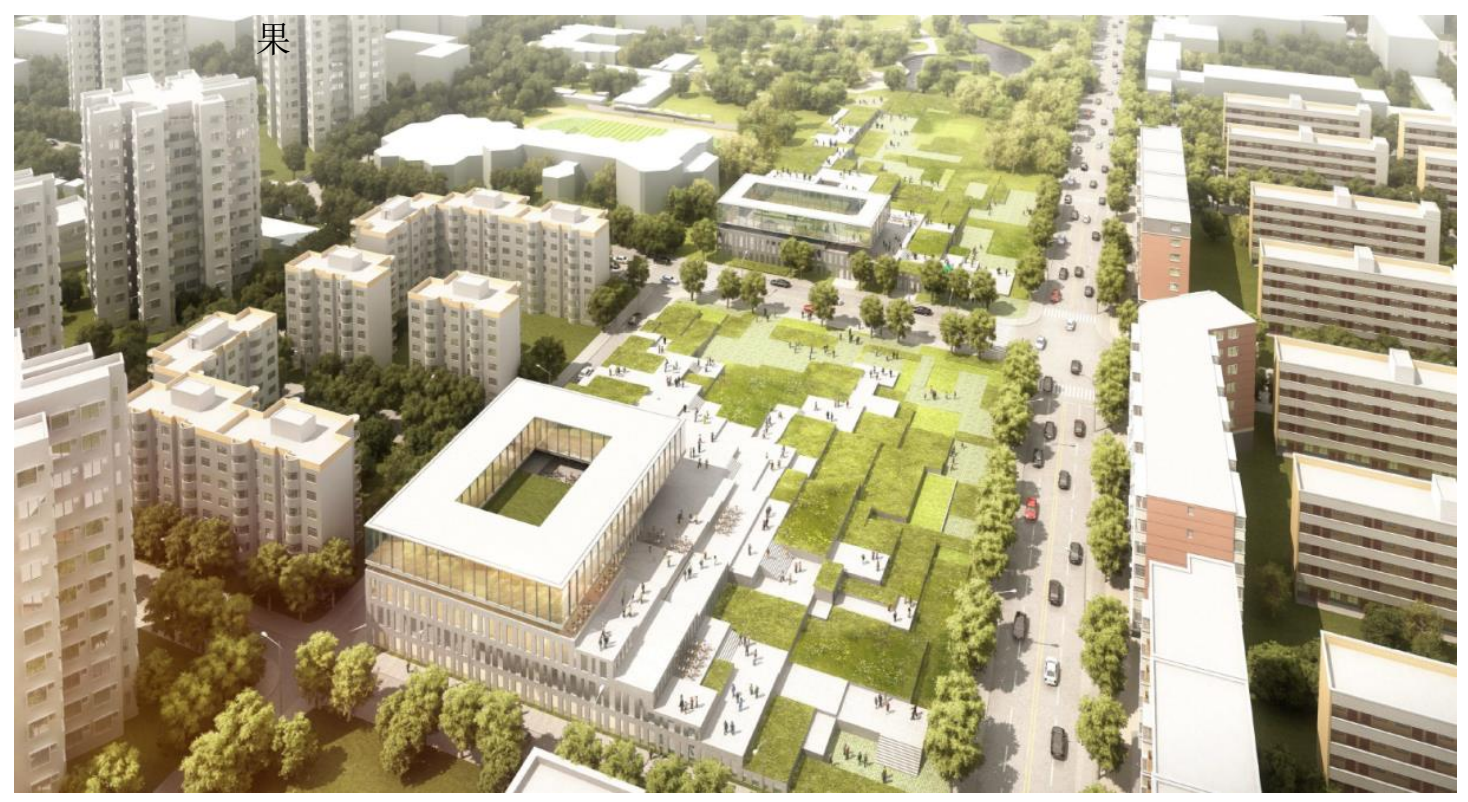

Figure 17 "Urban Green Step" community center plot effect
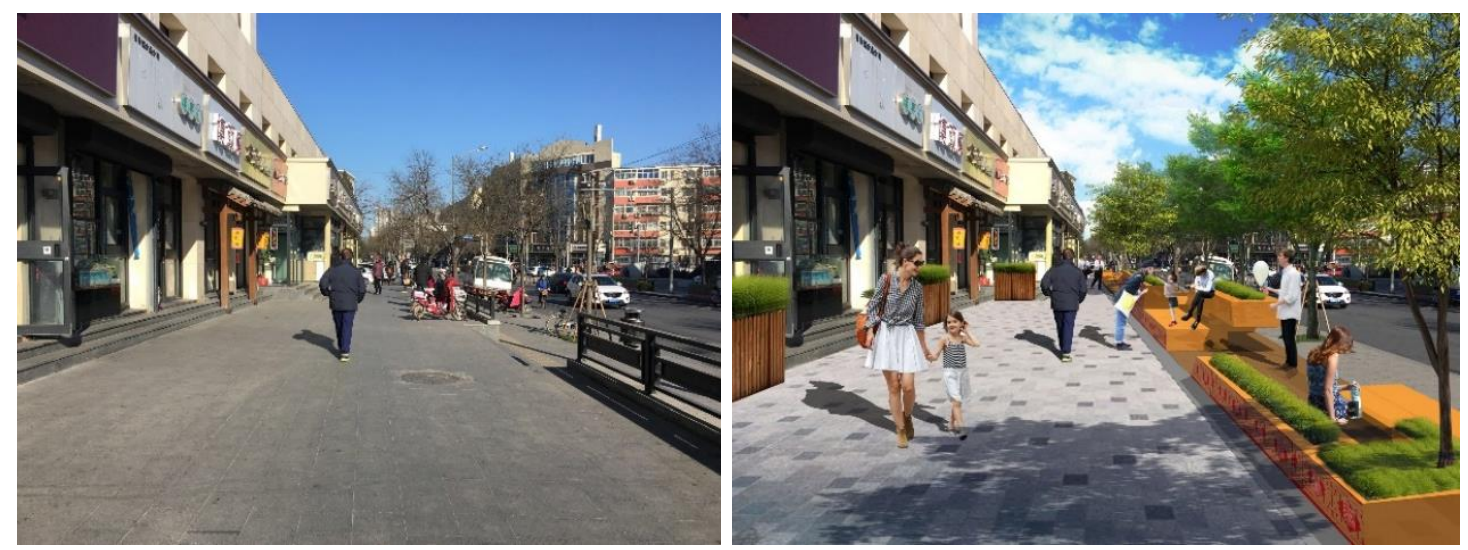

Fig. 18 Slow moving through flexible streets

(2) Strategy 2: Refine scene creation and optimize the quality of existing public space

In response to the deficiencies in the scale and quality of public space in the area as pointed out in the 
diagnostic report, this renewal plan follows the principles of focusing on the residents' usage needs and making full use of the current resources, avoiding major demolition and construction, and using microrenewal, scene creation and refined management to improve the quality of the existing street and activity space in the community. The main ways include: (1) Renovations of ground-level motor vehicle and non-motorized vehicle parking, unused debris piles and low-quality landscape flower beds on both sides of community streets. This is to create flexible roadside spaces with leisure, sports and interaction functions (Figure 18).

(2) Increase the scope of centralized activity space by demolishing old boiler houses and other illegal buildings, partially adjusting the internal roads of the district, and implanting fitness facilities, small courts, small restaurants, libraries, activity rooms, and other functions around public mini-plazas to form a clear internal community activity center (Figure 19); 3) fine-tuning and micro-renewal of building spacing space to improve non-motorized vehicle parking, temporary placement of household garbage, and neighborhood communication facilities.

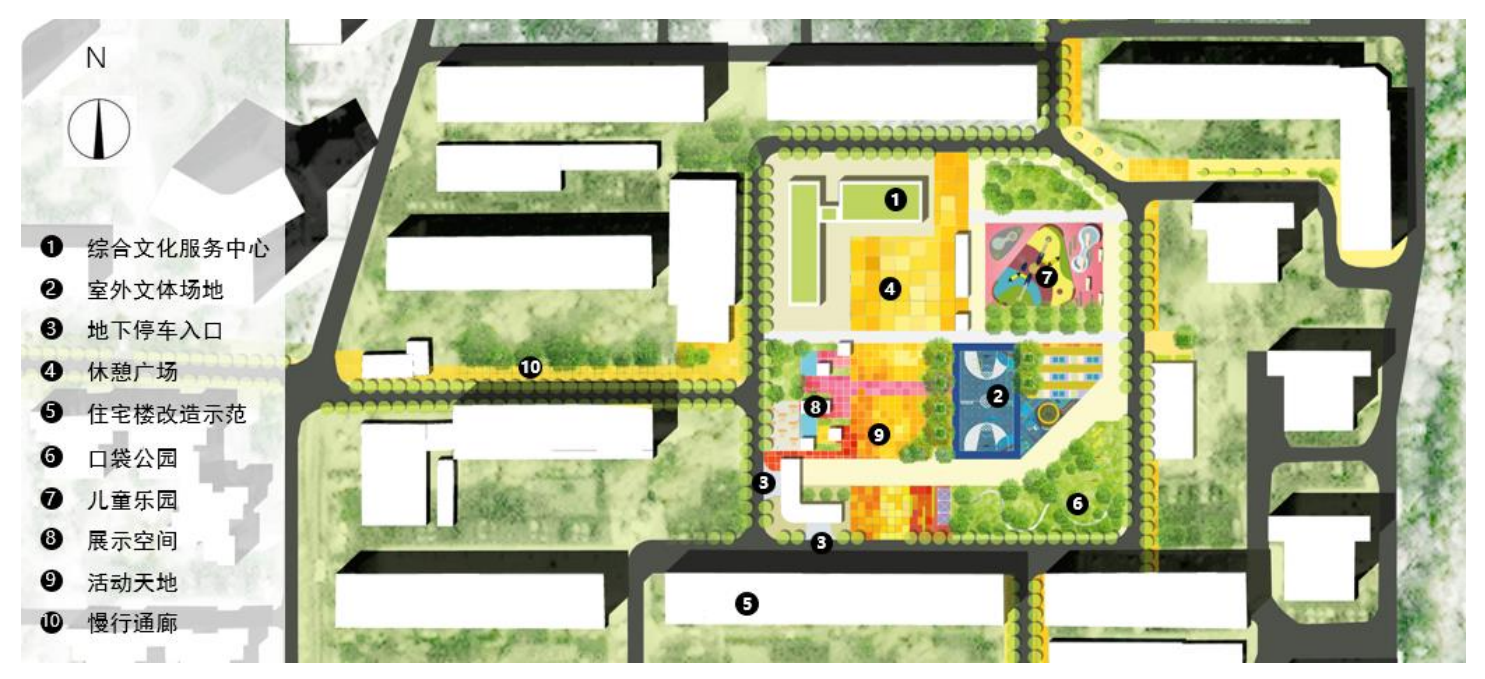

Figure 19 Community Internal Activity Center Design Master Plan

(3) Strategy 3: 15-minute living circle construction, complementing the basic public service facilities

The "15-minute community living circle" refers to the community area that provides basic services and public activities within a 15-minute walking distance of the residents' living location. It provides a safe, friendly, and comfortable platform for basic social and living functions. This concept was introduced in the Bajiao Community Renewal Plan to solve the problem of lack of supply in public services as revealed in the community urban diagnosis. The coverage of non-commercial facilities such as education, medical care and elderly care is calculated based on 15-minute walking distance (Figure 20), and the missing facilities are supplemented on this basis. For commercial facilities such as restaurants, cafes, supermarkets and culture, the demand for community activities in different time periods is firstly projected based on the results of urban inquiry, medical examination, and life system examination, and the supplementation of corresponding facilities is proposed accordingly. For commercial facilities such as restaurants, cafes, supermarkets, culture, etc., the demand for community activities at different times is first projected based on the results of the urban consultation, medical examination and life system examination. Based on this, the principles of supplementation, spatial support, and policy guidance are proposed accordingly.

\section{U isocarp}




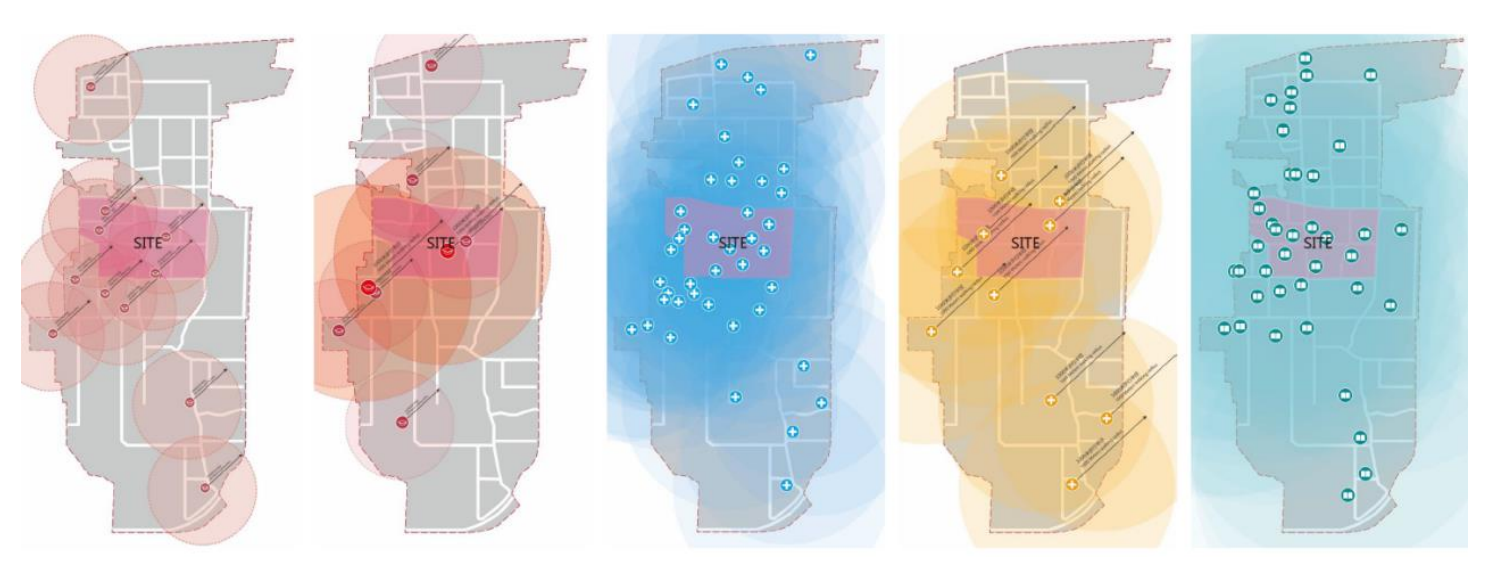

Figure 20 Planning of public service facilities in and around the community

(From left to right: kindergartens, primary and secondary schools, medical facilities, elderly facilities, cultural facilities)

\section{Conclusion}

Community is the basic unit of urban administration, an important support for non-productive urban activities, the basis for the quality of life and happiness of most residents, and an important guarantee of urban vitality and attractiveness. With the shift of China's urban development from increment-led to stock-led, the renewal of existing communities has become an important initiative to optimize urban quality and promote people's quality of life. The diverse crowd activities, historical data, and demands of existing communities determine the central position of accurate problem diagnosis in urban development, renewal and administration processes.

Based on the urban diagnostic approach, this paper uses multi-source big data and combines the actual situation and renewal needs of the Bajiao community to carry out diagnostic work including phenomena analysis, medical history research, urban inquiry and life system examination for the area, revealing the regional operation status and problems that cannot be reflected by traditional data and research methods. It provides a scientific basis for community renewal and guides the formulation of community renewal strategies and planning. As a micro-level practice of urban diagnosis, it has, to a certain extent, verified the practicality, advancement, and effectiveness of the urban diagnosis in understanding the operation status of cities, revealing urban development problems and their causes, and guiding the formulation of urban policies and plans.

\section{Note:}

(1)"big data, artificial intelligence, mobile internet, and cloud computing" are the new technologies represented by the big data, artificial intelligence, mobile internet, and cloud computing.

(2) Comprehensive urban diagnostic model is a diagnostic model based on the overall health status of the city under the integrated influence of various subsystems, specifically including city IQ model, urban vitality model, urban mobility model, and sustainable development model (Ye, Zhongnan, 2018).

(3) City Vitality Diagnostic Model is a comprehensive city diagnostic model that combines the characteristics of heat map and POI data, and introduces two indicators-crowd concentration intensity $Q$ and facility richness $D$, to describe the crowd concentration and public service supply in a certain time and space range, which is calculated as: 


$$
V_{r}=\left(\alpha \sum \mathrm{Q}_{\mathrm{rt}}+\beta D_{r}\right) / l
$$

Vr: The level of urban vitality within a radius $r$ of a location.

Qrt: the intensity of crowding within a radius $r$ at time $t$.

$$
\mathrm{t} \text { : time section, } \mathrm{t}=1,2,3, \ldots \ldots, 24 .
$$

Dr: Facility richness within a radius $r$.

r: radius of the study area.

$\alpha, \beta$ : indicator weights, determined by hierarchical analysis.

I: locational coefficient

(4) The reference standard in the table was established by selecting 15 similar areas (Shanghai Anshan New Village, Shanghai Caoyang New Village, Nanjing Gulou Community, etc.) across the country, and acquiring and comparing their corresponding data. The horizontal arrows indicate that the corresponding indicators are around the average value of the benchmark cases; upward arrows indicates that it is higher than the average value, and downward indicates that it is lower than the average value.

\section{References}

Ye, Z. (2018) Research on urban diagnosis based on big data [D]. Doctoral dissertation, Tongji University.

Beijing Institute of Surveying and Mapping Design. (2017) Evaluation report of urban physical examination of Bajiao Street, Shijingshan District, Beijing [R].

Luo, W. (2014) Smart City Diagnosis Diagnostic Assessment Model and Practice [M]. Beijing: People's Post and Telecommunications Publishing House.

Chai, Y., Liu, B., Liu, Y., et al. (2018) Construction and calculation of urban physical diagnostic index based on multi-source big data - taking Shanghai as an example [J]. Geoscience, 38(1):1-10.

Xu, Q., He, Y., Gan, L., et al. (2018) From urban physical examination to neighborhood diagnosis--a study on urban renewal in Dajie bar [J]. Beijing Planning and Construction, (2): 142-148.

Ye, Z. (2016) Quantification and Diagnosis of Urban Mobility - A Study of Methodology Based on Network Map Data and Accessibility Model [J]. Southern Architecture, (5): 66-70.

$\mathrm{Li}, \mathrm{M}$. (2017) Research on the planning measures of "15-minute community living circle" based on the characteristics of residents' behavioral needs[J]. Journal of Urban Planning, (1): 111-118. 